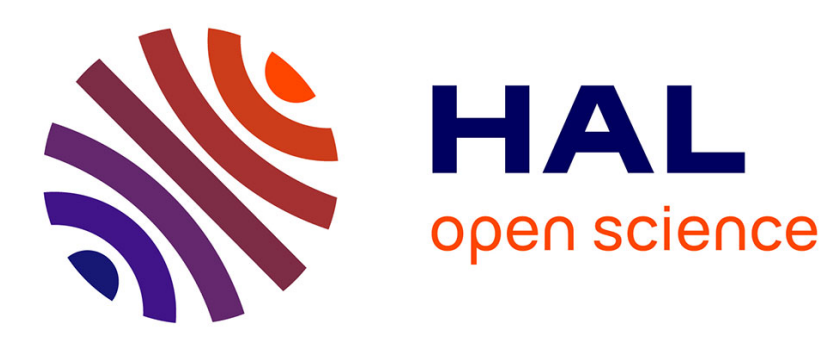

\title{
Local order of orthorhombic weberite-type Y3TaO7 as determined by neutron total scattering and density functional theory calculations
}

Igor Gussev, Eric O'Quinn, Gianguido Baldinozzi, Jörg Neuefeind, Rodney Ewing, Fuxiang Zhang, Maik Lang

\section{To cite this version:}

Igor Gussev, Eric O'Quinn, Gianguido Baldinozzi, Jörg Neuefeind, Rodney Ewing, et al.. Local order of orthorhombic weberite-type $\mathrm{Y} 3 \mathrm{TaO} 7$ as determined by neutron total scattering and density functional theory calculations. Acta Materialia, inPress, 10.1016/j.actamat.2020.07.005 . hal-02898876

\section{HAL Id: hal-02898876 https://hal.science/hal-02898876}

Submitted on 14 Jul 2020

HAL is a multi-disciplinary open access archive for the deposit and dissemination of scientific research documents, whether they are published or not. The documents may come from teaching and research institutions in France or abroad, or from public or private research centers.
L'archive ouverte pluridisciplinaire HAL, est destinée au dépôt et à la diffusion de documents scientifiques de niveau recherche, publiés ou non, émanant des établissements d'enseignement et de recherche français ou étrangers, des laboratoires publics ou privés. 


\section{Journal Pre-proof}

Local order of orthorhombic weberite-type $\mathrm{Y}_{3} \mathrm{TaO}_{7}$ as determined by neutron total scattering and density functional theory calculations

Igor M. Gussev, Eric C. O'Quinn, Gianguido Baldinozzi , Jörg Neuefeind, Rodney C. Ewing, Fuxiang Zhang, Maik Lang

PII:

DOI:

Reference:

To appear in:

Received date:

Revised date:

Accepted date:
S1359-6454(20)30503-6

https://doi.org/10.1016/j.actamat.2020.07.005

AM 16145

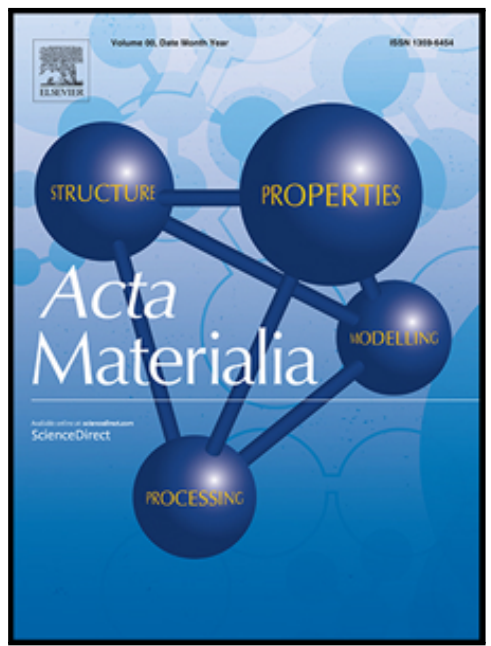

Please cite this article as: Igor M. Gussev, Eric C. O'Quinn, Gianguido Baldinozzi, Jörg Neuefeind, Rodney C. Ewing, Fuxiang Zhang, Maik Lang, Local order of orthorhombic weberite-type $\mathrm{Y}_{3} \mathrm{TaO}_{7}$ as determined by neutron total scattering and density functional theory calculations, Acta Materialia (2020), doi: https://doi.org/10.1016/j.actamat.2020.07.005

This is a PDF file of an article that has undergone enhancements after acceptance, such as the addition of a cover page and metadata, and formatting for readability, but it is not yet the definitive version of record. This version will undergo additional copyediting, typesetting and review before it is published in its final form, but we are providing this version to give early visibility of the article. Please note that, during the production process, errors may be discovered which could affect the content, and all legal disclaimers that apply to the journal pertain.

(C) 2020 Published by Elsevier Ltd on behalf of Acta Materialia Inc. 
Local order of orthorhombic weberite-type $\mathrm{Y}_{3} \mathrm{TaO}_{7}$ as determined by neutron total scattering and density functional theory calculations

Igor M. Gussev $^{1}$, Eric C. O’Quinn ${ }^{1}$, Gianguido Baldinozzi ${ }^{2}$, Jörg Neuefeind ${ }^{3}$, Rodney C. Ewing ${ }^{4}$, Fuxiang Zhang ${ }^{5}$, and Maik Lang ${ }^{1, *}$

1 Department of Nuclear Engineering, University of Tennessee, Knoxville, TN, 37996, USA

2 Laboratoire Structures, Propri'et'es et Mod'elisation des Solides, CNRS, CentraleSup'elec, Universit’e Paris-Saclay, 91190 Gif-sur-Yvette, France

3 Chemical and Engineering Materials Division, Spallation Neutron Source, Oak Ridge National Laboratory, Oak Ridge, TN, 37831

4 Department of Geological Sciences, Stanford University, Stanford, CA, 94305, USA

5 Materials Science and Technology Division, Oak Ridge national Laboratory, Oak Ridge, TN 37831, USA

* Corresponding author: Dr. Maik Lang

Email address: mlang2@utk.edu

Phone: +1 8659742525

Address: 206 Nuclear Engineering Building, 1412 Circle Dr, Knoxville, TN 37916 
This work was supported by the U.S. Department of Energy, Office of Science, Basic Energy Sciences, under Award DE-SC0020321. 


\section{Abstract:}

Weberite-type oxides are a family of oxides with $\mathrm{A}_{3} \mathrm{BO}_{7}$ stoichiometry that can adopt different space groups depending on chemical composition. There is a discrepancy in previous studies as to whether $\mathrm{Y}_{3} \mathrm{TaO}_{7}$ is the orthorhombic $C 222_{1}$ or the $C c m m$ space group. Here, we describe the short- and long-range structural properties of weberite-type $\mathrm{Y}_{3} \mathrm{TaO}_{7}$ using neutron total scattering data, which has a high sensitivity to the oxygen sublattice. Simultaneous analysis of both shortand long-range structural data via conventional Rietveld and small box modelling demonstrates that $\mathrm{Y}_{3} \mathrm{TaO}_{7}$ is best modeled as $C 222_{1}$. While the $C c m m$ describes equally well the long-range structure, pair distribution function analysis revealed that the local atomic configuration can be best modelled as $C 222_{1}$. This is corroborated by first-principles calculations that confirm the energetic preference of the $C 222_{1}$ over $C \mathrm{cmm}$. Neutron total scattering data are reported for the first time for the $\mathrm{Y}_{3} \mathrm{TaO}_{7}$ weberite-type ceramics.

Keywords: Neutron diffraction, Pair distribution function analysis, Density functional theory, Crystalline oxides, Rare-earth 


\section{Introduction:}

Advances in the development of energy technologies require specifically engineered materials that maintain structural stability in harsh working conditions, such as extreme radiation fields and high temperatures [1]. Complex oxide ceramics are promising candidates for a number of energy-related applications, such as nuclear waste-containing matrices and solid electrolyte fuel cells. Despite the wide body of research into the radiation response of such materials [2]-[5], there is a limited understanding of the impact of extreme environments on their atomic-scale behavior. Recent neutron total scattering experiments have revealed that the local structure plays an important role in understanding irradiation-induced structural disorder in pyrochlore oxides $\left(\mathrm{A}_{2} \mathrm{~B}_{2} \mathrm{O}_{7}\right)$ [5]. Depending on the chemical composition, ion irradiation will either induce an order-to-disorder transformation (all the while maintaining long-range crystallinity) or lead to complete amorphization [6], [7]. Analysis of neutron total scattering revealed that the local atomic arrangement is nearly identical in both amorphous and disordered pyrochlore oxides and can be best described by a weberite-type structural model [8]. Intriguingly, this weberite-type atomic arrangement also has been reported for pyrochlore compositions disordered by a variety of other methods including high-temperature treatment, high-energy ball milling [9], chemical doping, or deviation from ideal stoichiometry [10]. As this weberite-type configuration appears to be a general phenomenon in disordered pyrochlore, there has recently been an increased interest [11] in oxide materials that exhibit the weberite-type structure over all length-scales.

Weberite-type tantalates (general formula $\mathrm{A}_{3} \mathrm{BO}_{7}$ ) with the A-site occupied by a rare-earth cation and the B-site occupied by Ta, can display either an ordered, orthorhombic weberite-type structure or a disordered, anion-deficient fluorite structure. The size of the A-site cation determines whether an ordered or disordered phase is formed. When the A-site cation is large 
(La, Ce, Pr) weberite-type oxides adopt a $\mathrm{Cmcm}$ space group, while medium-sized (Y, Pm through Dy) and smaller cations (Er through $\mathrm{Lu})$ are orthorhombic $\left(C 222_{1}\right)$ and the disordered, anion-deficient fluorite $(F m-3 m)$ structures, respectively [12]. $\mathrm{Nd}_{3} \mathrm{TaO}_{7}$ can be synthesized as a mixed-phase material $\left(\mathrm{Cmcm}\right.$ and $\left.\mathrm{C} 222_{1}\right)$, while $\mathrm{Ho}_{3} \mathrm{TaO}_{7}$ can form either a $C 222_{1}$ or $\mathrm{Fm}$-3m phase, depending on the synthesis conditions and temperature treatment. [12]. There is no consensus in the literature [13], [14] on the best structural model for $\mathrm{Y}_{3} \mathrm{TaO}_{7}$ and different space groups have been proposed, including C222 ${ }_{1}$ [11], [12], [15], [16], Ccmm [14] and Fm-3m [17]. Previous neutron total scattering experiments showed that the $\mathrm{C} 222_{1}$ space group was the best fit to the local atomic arrangement of $\mathrm{Yb}_{3} \mathrm{TaO}_{7}$ with a long-range disordered-fluorite structure [18]. Most of these studies utilized either X-ray diffraction with limited structural information on the oxygen sublattice and local atomic arrangements or Raman spectroscopy that yields complex vibrational information, particularly for low-symmetry structures.

In this study, the limitations of X-ray diffraction and Raman spectroscopy are overcome by utilizing neutron total scattering. The weberite-type $\mathrm{Y}_{3} \mathrm{TaO}_{7}$ has favorable elements for neutron scattering analysis with $\mathrm{Y}$ having a large neutron scattering cross section and low absorption cross-section. Neutron total scattering simultaneously provides information on both short- and long-range structures with high sensitivity to the oxygen sublattice. Here we report, using analysis of diffraction data and pair distribution functions, that the orthorhombic $C 222_{1}$ is the most appropriate structural model for the $\mathrm{Y}_{3} \mathrm{TaO}_{7}$ weberite-type compound. The validity of the proposed structural model is supported by density functional theory (DFT) calculations as energetically the most stable configuration. 


\section{Experimental Methods:}

\subsection{Sample synthesis}

Polycrystalline powder of $\mathrm{Y}_{3} \mathrm{TaO}_{7}$ was synthesized using a solid-state reaction process. Powders of tantalum oxide $\left(\mathrm{Ta}_{2} \mathrm{O}_{5}\right)$ and yttrium oxide $\left(\mathrm{Y}_{2} \mathrm{O}_{3}\right)$ with a purity of $99.9998 \%$ were used as starting materials. The powders were preheated in air at $1000 \mathrm{~K}$ for $12 \mathrm{~h}$ to remove any moisture and subsequently mixed, ground, and pressed into pellets. These pellets were heated in air at $1200 \mathrm{~K}$ for $48 \mathrm{~h}$, reground and pressed again into pellets and reheated in air at $1500 \mathrm{~K}$ for $96 \mathrm{~h}$.

\subsection{Neutron Total Scattering Analysis}

The as prepared sample pellet was reground into a fine powder, which was then loaded into a 2 $\mathrm{mm}$ quartz capillary with a wall thickness of $0.01 \mathrm{~mm}$. Neutron total scattering characterization was performed at the Nanoscale-Ordered Materials Diffractometer (NOMAD) beamline BL-1B at the Spallation Neutron Source at Oak Ridge National Laboratory [19]. The measurement was performed at room temperature with an exposure time of 64 minutes. The six NOMAD detector banks were calibrated using scattering from a diamond powder and silicon sample. The silicon standard sample was individually measured and used to obtain the instrument parameter file for the Rietveld refinements. Scattering intensity of measured sample was normalized to the scattering intensity of a solid vanadium rod to get the correct scaling parameters for the structure function of the sample. The scattering intensity from an empty sample position as well as from an empty $2 \mathrm{~mm}$ quartz capillary were measured as background signal and automatically subtracted from the scattering intensity of the polycrystalline powder samples.

A Fourier transform was applied to the structure functions, $\mathrm{S}(Q)$, to obtain the pair distribution function, $\mathrm{G}(r)$ : 


$$
\mathrm{G}(\mathrm{r})=A \int_{\mathrm{Q}_{\min }}^{\mathrm{Q}_{\max }} \mathrm{Q}(\mathrm{S}(\mathrm{Q})-1) \operatorname{Sin}(\mathrm{Qr}) \mathrm{dQ}
$$

with maximum momentum transfer $\left(Q_{\max }\right)$ of $31.42 \AA^{-1}$. A is the arbitrary scale parameter. The momentum transfer is defined as the following:

$$
\mathrm{Q}=4 \pi \sin \theta / \lambda
$$

where $\lambda$ and $\theta$ are the neutron wavelength and scattering angle, respectively.

\subsection{Long-range data refinement}

The long-range structure was analyzed using Rietveld refinement [20] performed in the GSAS package [21]. The set of highest Q-resolution backscattering detector banks was used in the refinement. The $C 222_{1}$ phase of $\mathrm{Y}_{3} \mathrm{TaO}_{7}$ was refined using 33 parameters: unit cell parameters $(a, b, c)$, scale factor, 6 background parameters (Shifted Chebyshev function type), 6 positional parameters for cations, 9 positional parameters for anions and a total of 8 Uiso parameters for all of the sites. Similarly, the Ccmm phase of $\mathrm{Y}_{3} \mathrm{TaO}_{7}$ was refined using 26 parameters: unit cell parameters $(a, b, c)$, scale factor, 6 background parameters, 2 positional parameters $(\mathrm{x} \neq \mathrm{y})$ for $8 \mathrm{~g}$ site, 3 positional parameters $(\mathrm{x} \neq \mathrm{y} \neq \mathrm{z})$ for $16 \mathrm{~h}$ oxygen site, 3 positional parameters for three $4 \mathrm{c}$ oxygen sites (x) and a total of 8 Uiso parameters for all of the sites. Sets of diffraction data from three detector banks (3,4 and 5) with different space coverage and resolution [19] were refined simultaneously.

\subsection{Short-range data refinement}

Small-box refinement of pair distribution functions (PDF) representing the short-range structure was performed using PDFgui software [22]. The $C 222_{1}$ phase of $\mathrm{Y}_{3} \mathrm{TaO}_{7}$ was refined using 43 parameters: unit cell parameters $(a, b, c)$, the scale factor, the delta2 factor, which is a PDFgui 
specific correlated motion factor used in conjunction with thermal parameters, 5 positional parameters for the cations ( $x$ for $4 a$ site, $y$ for $4 b$ site, $x \neq y \neq z$ for $8 \mathrm{c}$ site), 4 harmonic thermal displacement parameters $\left(u^{11} u^{22} u^{33}\right.$ and $\left.u^{13}\right)$ for each of the $4 b$ sites and 6 for the $8 c$ site, 9 positional parameters for the anions (three sets of $\mathrm{x}$ for $4 \mathrm{a}$ site, and 2 sets of $(\mathrm{x} \neq \mathrm{y} \neq \mathrm{z})$ for $8 \mathrm{c}$ sites), 6 harmonic $\mathrm{u}^{\mathrm{ij}}$ thermal displacement parameters for the $8 \mathrm{c}$ sites, 4 harmonic thermal displacement parameters $\left(\mathrm{u}^{11} \mathrm{u}^{22} \mathrm{u}^{33}\right.$ and $\mathrm{u}^{23}$ ) for each of the $4 \mathrm{~b}$ sites. The Ccmm phase of $\mathrm{Y}_{3} \mathrm{TaO}_{7}$ was refined using 34 parameters: unit cell parameters $(a, b, c)$, the scale factor, the delta2 factor, 2 positional parameters for the cations $(x \neq y$ for $8 g$ site $)$ and 9 harmonic $\left(u^{11} \neq u^{22} \neq\right.$ $\left.u^{33}\right)$ thermal parameters, 6 positional parameters used for anions(set of $3(x \neq y \neq z)$ for $16 h$ oxygen sites and $3 \mathrm{x}$ for three $4 \mathrm{c}$ oxygen sites). Every oxygen $4 \mathrm{c}$ site had an individual set of three harmonic $\left(\mathrm{u}^{11} \neq \mathrm{u}^{22} \neq \mathrm{u}^{33}\right)$ thermal parameters, the 16 h site had 6. Partial PDF's are produced from the calculation of individual interatomic distances from the resultant PDF structure using the calculation tool built-in the PDFgui software.

\subsection{Density functional theory}

First principles calculations were used to evaluate relative stabilities of the two orthorhombic phases. These electronic structure calculations were performed within the DFT framework using the projector augmented wave (PAW) approach for the core-valence interaction and the general gradient approximation with Perdew-Burke-Ernzerhof (PBE) [23] generalized gradient approximation for the exchange-correlation functional as implemented in the VASP code [24].The potentials included 11 valence electrons for Ta, 11 for $\mathrm{Y}$, and 6 for O. A plane-wave kinetic energy cutoff was determined at $520 \mathrm{eV}$, with a uniform k-point mesh $(6 \times 6 \times 5)$ for Brillouin zone sampling based on the Monkhorst-Pack scheme that was found to be sufficient to achieve well-converged energies. These parameters were used for static self-consistent 
calculations of the primitive cells having $\mathrm{Cmcm}$ and $\mathrm{C} 222_{1}$ symmetries respectively. Electronic self-consistency was considered to be achieved when the total energy change between electronic steps was less than $1 \mu \mathrm{eV}$. The lattice metric was relaxed for all crystal symmetries and the internal structural parameters were relaxed until all Hellmann-Feynman forces on each ion were less or equal $2 \mathrm{meV} / \AA$. $C m c m$ and $C 222_{1}(b, a,-c)$ structures acquired from the DFT calculations were later transposed into $C \mathrm{cmm}$ and $C 222_{1}(a, b, c)$ via simple lattice vector exchange for comparison with experimental results.

\section{Results and Discussion:}

Refinements of neutron diffraction data confirmed that the as-prepared $\mathrm{Y}_{3} \mathrm{TaO}_{7}$ material was a pure single phase. The diffraction patterns (NOMAD detector banks 3 through 5) could not be indexed with a disordered, anion-deficient fluorite $(\mathrm{Fm}-3 \mathrm{~m})$ phase due to the obvious presence of peaks not indexed by the $F m-3 m$ space group. The refinements with the two orthorhombic structural models proposed in literature, $\mathrm{Ccmm}$ and $C 222_{1}$, are shown in Figure 1. These refinements (Table 1) revealed that both orthorhombic models yield nearly identical refinement quality as expressed by the goodness-of-fit parameter $R_{w}(8.84 \%$ and $8.75 \%$ for $C \mathrm{cmm}$ and $C 222_{1}$, respectively).The two orthorhombic structural models acquired from the Rietveld refinements (Figure 2) are similar in terms of symmetry, and they are related to the cubic fluorite structure. The idealized orthorhombic structure (Figure 2a) consist of 6-coordinated Ta cations forming octahedra $\left(\mathrm{TaO}_{6}\right)$ as well as 8 and 7-coordinated $\mathrm{Y}$ cations forming distorted cubes $\left(\mathrm{YO}_{8}\right.$ and $\mathrm{YO}_{7}$, where one oxygen site is replaced by a vacant site). The $\mathrm{TaO}_{6}$ octahedra are tilted toward [100], share one oxygen atom, and form an infinite one-dimensional zig-zag chain projected along the [001]. The $\mathrm{YO}_{8}$ distorted cubes share edges and form chains parallel to [001]. 
Combinations of those two chains form slabs parallel to (011); whereas the $\mathrm{YO}_{7}$ distorted cubes are located between these slabs.

Although both orthorhombic structure types share the same cation sublattice, there is a difference in symmetry of the anion sublattice, as shown in Figures $2 b$ and $2 c$. Oxygen atoms located at the $16 h$ Wyckoff site in the Ccmm structure are split into two identical $8 c$ Wyckoff sites in the C222 structure. The latter allows for subtle distortions of the 6-coordinated Ta polyhedron, as well as the 7- and 8-coordinated yttrium polyhedra. This effect leads to slight variations in the $\langle\mathrm{O}-\mathrm{O}\rangle$ interatomic distances among both structure types. Although these variations are very small, they impact Rietveld refinement of the diffraction patterns and have an influence on the goodness-offit parameter $R_{w}$. This value is slightly lower for the $C 222_{1}$ model (Figure 1), which indicates that this structure-type fits the data somewhat better. Application of a significance test developed by Hamilton [25] results in a confidence value of $99.95 \%$ that $\mathrm{C} 222_{1}$ is a more appropriate structural model, despite the addition of several refinement parameters. This similarity in $\mathrm{R}$ factors of both structural models explains the discrepancy in literature [12]-[17] and highlights that diffraction experiments alone, even with neutron probes, are most likely not unambiguous for the accurate determination of the structure of $\mathrm{Y}_{3} \mathrm{TaO}_{7}$

Insight into the short-range structure was obtained through analysis of the pair distribution function (Figure 3). Small-box refinements based on the Ccmm and $C 222_{1}$ structural models yield $R_{w}$ values of $7.18 \%$ and $5.54 \%$ for $C \mathrm{cmm}$ and $C 222_{1}$, respectively (Table 1). As indicated by the difference curves of data and model fit, the higher $R_{w}$ value for $C \mathrm{cmm}$ is primarily related to deviations in intensity and position of the peak at $2.8 \AA$, denoted by an asterisk. The origin of this behavior can be better seen in the PDF data capturing only the $r$-range of local coordination polyhedra with the $\langle\mathrm{Ta}-\mathrm{O}\rangle,\langle\mathrm{Y}-\mathrm{O}\rangle$, and $\langle\mathrm{O}-\mathrm{O}\rangle$ nearest neighbor distances (Figure 4). The 
partial PDFs reveal that the $\langle\mathrm{O}-\mathrm{O}\rangle$ interatomic correlations are responsible for the peak in the PDF data around $2.8 \AA$. The $16 h$ Wyckoff oxygen site is too constrained by symmetry in the Ccmm structural model to fully capture the pair correlations of the major coordination polyhedra. The $\langle\mathrm{O}-\mathrm{O}\rangle$ nearest neighbor correlations in the experimental PDF data are broader and shifted to larger $r$-values (Figure 4). This is better captured by the $C 222_{1}$ structural model, which allows for less symmetric oxygen arrangement due to the presence of two $8 c$ oxygen sites. While overall both structural models fit the data very well across the short-range, this small difference related to the oxygen sublattice indicates that the $C 222_{1}$ structural model is indeed most appropriate for the weberite-type $\mathrm{Y}_{3} \mathrm{TaO}_{7}$. Only a local probe with high sensitivity to the oxygen sublattice is able to capture the actual atomic arrangement as demonstrated in this study by neutron total scattering utilizing the pair distribution function analysis. Thus, the present neutron total scattering study resolves the discrepancy in literature and confirms the validity of the $C 222_{I}$ structural model from the local atomic arrangement to the long-range behavior. This also in agreement with physical properties measurements of ordered weberite-type oxides [11], [12]. The occurrence of a second harmonic in laser-related applications can only be understood by distortions of the Ta polyhedron and the absence of an inversion center as required by the $C 222_{1}$ symmetry.

Density functional theory (DFT) modeling was employed to gain further insight into the energetic stability of both orthorhombic structural models for $\mathrm{Y}_{3} \mathrm{TaO}_{7}$ (structural parameters are given in Table 2). The DFT results revealed an energy value at $0 \mathrm{~K}$ of $-207.0408 \mathrm{eV}$ and $207.1525 \mathrm{eV}$, for $\mathrm{Cmcm}$ and $\mathrm{C} 222_{1}$, respectively. This confirms that the atomic configuration with the lowest energy state for $\mathrm{Y}_{3} \mathrm{TaO}_{7}$ is best described with the $C 222_{1}$ structural model in agreement to the neutron PSF results. The difference between the cohesive energies at $0 \mathrm{~K}$ is 
significant though small enough to consider a possible phase transition to the centrosymmetric structure at high temperature. A comparison of the $C 222_{1}$ structure from DFT modelling and experimental results from small-box analysis reveals small deviations between experiment and simulation While the backbone of the structure, the 6-coordinated Ta polyhedra, is identical, the 8-coordinated Y polyhedra are slightly shifted against each other within the reference frame. This offset leads to a small difference that is only visible along the [001] in the weberite-type unit cell in the DFT model, which is compensated for by an increased bond distance of the two weakly bound oxygen atoms away from the 8-coordinated cation. This elongates the 8coordinated polyhedral parallel to [100] and changes their size (larger in the DFT model) inducing a more pronounced zig-zag type pattern of the $\mathrm{Y}$ polyhedra in the DFT structure observed along [001].

The small discrepancy between the structural model from DFT calculations and PDF refinements can be explained by the absence of thermal effects in the calculation (performed at $0 \mathrm{~K}$ ). In addition, the [100] and [001] lattice dimensions are more rigid in terms of electronic structure and the system compensates for any distortion or rotation of polyhedra by adjusting the "soft" dimension of the electronic structure, which is [010] or the b-lattice parameter.

\section{Conclusion:}

Weberite-type $\mathrm{Y}_{3} \mathrm{TaO}_{7}$ was investigated using neutron total scattering analysis by the means of Rietveld refinement, small-box modelling and by applying first-principles calculations. Neutron total scattering with high sensitivity to the oxygen sublattice along with simultaneous access to short- and long-range structures identify the $C 222_{1}$ space group as most appropriate structural model. While the long-range structure shows only subtle differences between $C 222_{1}$ and $C \mathrm{cmm}$, the local structure is much better described by the $C 222_{1}$ structural model. The only difference 
between both orthorhombic structural models is within the local atomic arrangement of the anion sublattice, which is inaccessible by conventional X-ray diffraction experiments. These experimental results are corroborated by DFT calculations that show that the $C 222_{1}$ structural model is energetically more stable $(-207.1525 \mathrm{eV})$ as compared with the Ccmm model (207.0408 eV). These results may also impact the local weberite-type ordering that was recently reported for disordered pyrochlore oxides. 


\section{Acknowledgments:}

This work was supported by the U.S. Department of Energy, Office of Science, Basic Energy Sciences, under Award DE-SC0020321. E.C.O. acknowledges support from the U.S. Department of Energy, Office of Science, Office of Workforce Development for Teachers and Scientists, Office of Science Graduate Student Research (SCGSR) program. The SCGSR program is administered by the Oak Ridge Institute for Science and Education for the DOE under contract number DESC0014664. The research at ORNL's Spallation Neutron Source was sponsored by the Scientific User Facilities Division, Office of Basic Energy Sciences, U.S. Department of Energy. We thank Michelle Everett (ORNL) for guidance during the neutron scattering experiments. 


\section{Figure and Tables}

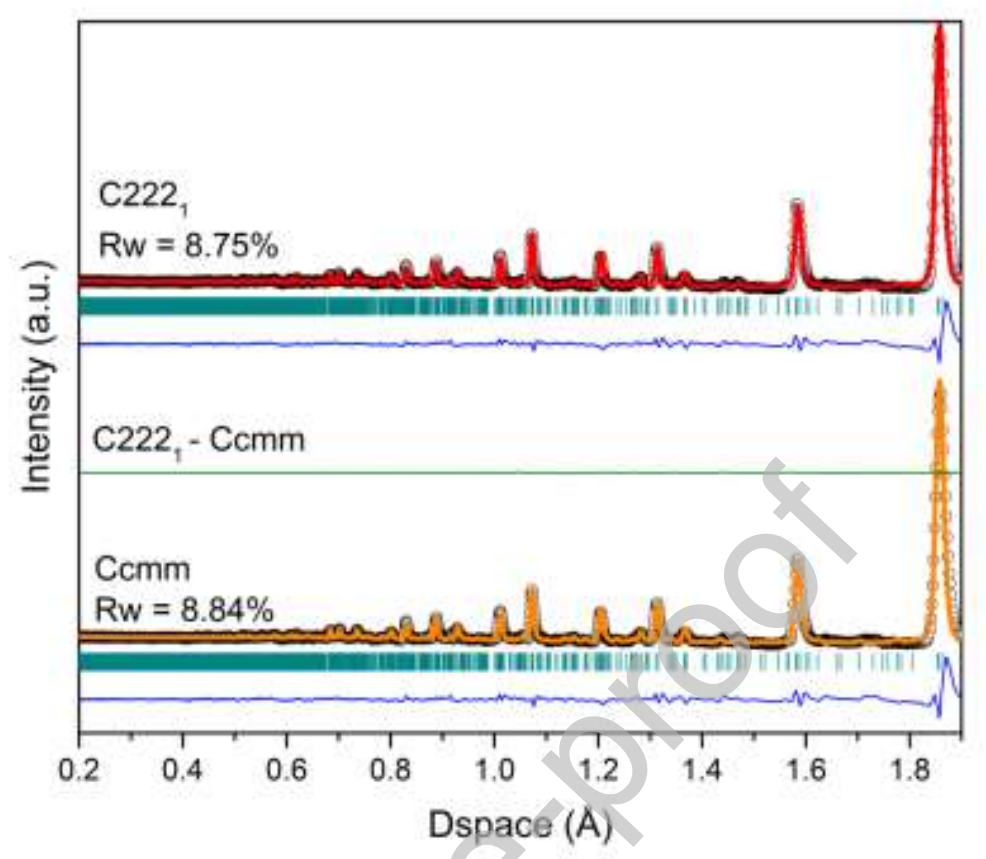

Figure 1: Neutron diffraction pattern of $\mathrm{Y}_{3} \mathrm{TaO}_{7}(\mathrm{NOMAD}$ detector bank 4) as measured (black circles) and modeled using $C \mathrm{cmm}$ (orange line, bottom profile) and $C 222_{1}$ (red line, top profile) space groups. The blue lines represent the difference curves between the experimental data and the model fit with the $R_{\mathrm{w}}$ values being the goodness-of-fit parameter. The green line represents the difference in diffraction profiles between the $C 222_{l}$ and $C c m m$ model, respectively. 


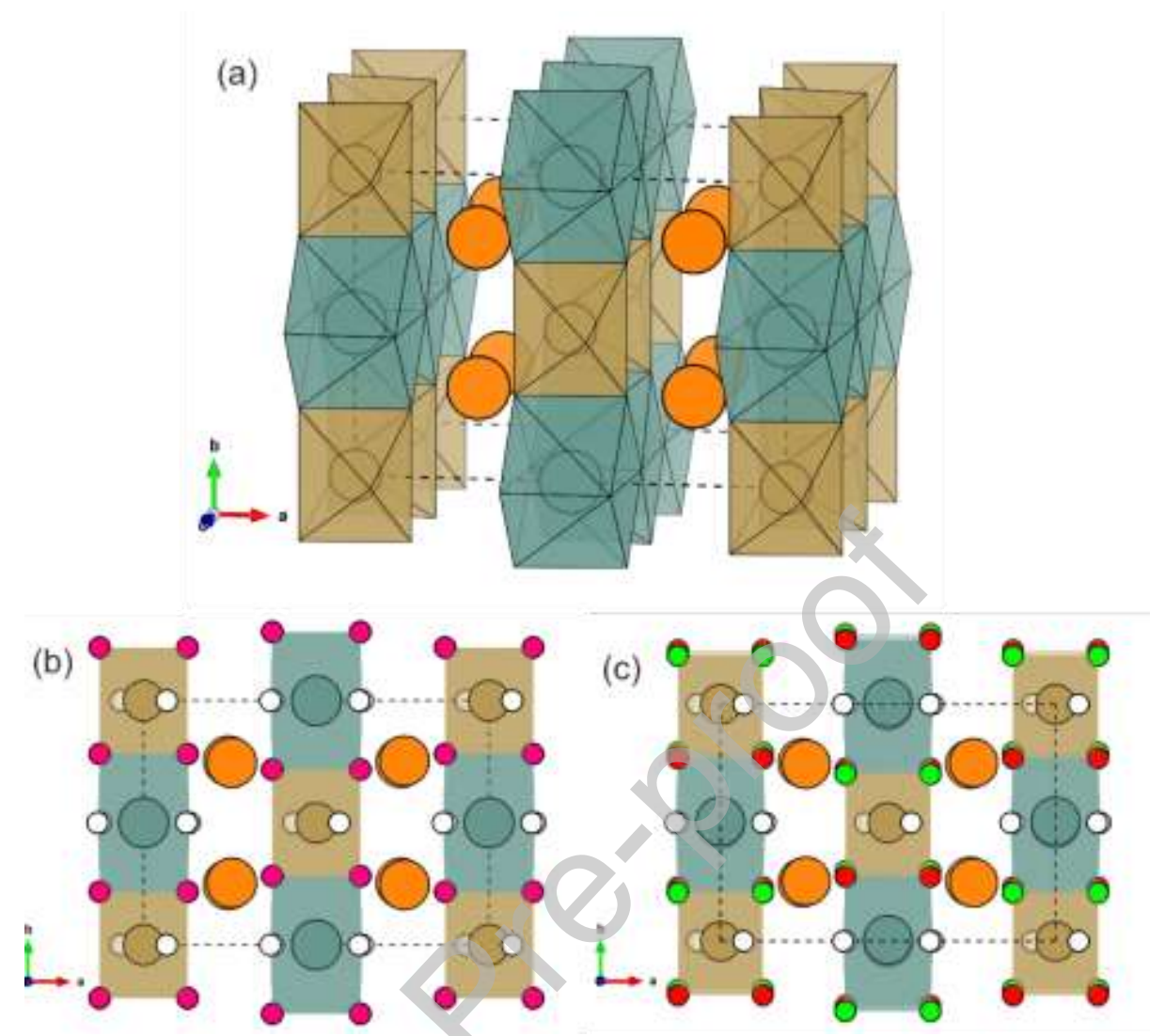

Figure 2: Schematic representations of the $\mathrm{Y}_{3} \mathrm{TaO}_{7}$ structures with dashed black lines representing the unit cells. (a) Idealized structural model with cations are shown for tantalum as golden-sand colored spheres and yttrium as teal color spheres (8-coordinated) or as orange spheres (7-coordinated). Selected coordination polyhedra are represented using similar shades ( $\mathrm{YO}_{7}$ polyhedra are not shown). (b) Structure resulting from GSAS refinement using Ccmm symmetry viewed along [001]. 16h oxygen sites are shown as smaller magenta spheres, while three 4a oxygen sites are displayed as white spheres. (c) Structure resulting from GSAS refinement using $C 222_{1}$ symmetry viewed along [001]. Two 8c oxygen sites are drawn as red and green spheres while three $4 \mathrm{a}$ oxygen sites are shown as white spheres. The $\mathrm{C} 222_{1}$ structure is shifted by $1 / 4 c$ along [001] for the ease of comparison. 


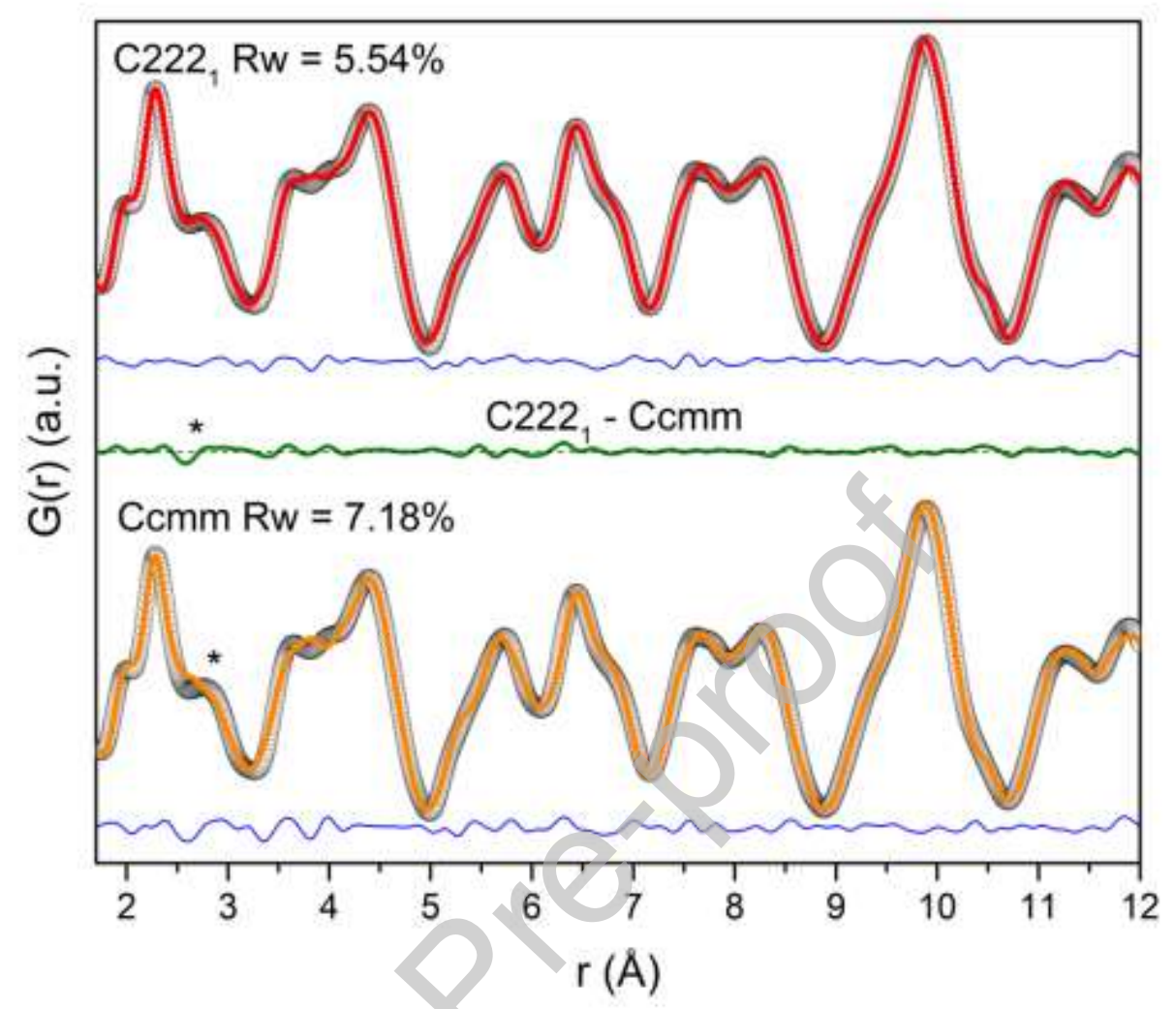

Figure 3: Neutron pair distribution functions (PDF) of $\mathrm{Y}_{3} \mathrm{TaO}_{7}$ modeled with $C \mathrm{cmm}$ and $C 222_{1}$ space groups (bottom and top profiles, respectively). Black circles represent the experimental data and red and orange lines are model fits. The blue lines are difference curves between data and the model and the green line is the relative difference between the $C 222_{1}$ and $C \mathrm{cmm}$ model fits. The asterisk (*) at $2.8 \AA$ in bottom figure indicates the deviation between experimental data and refinement with the $C c m m$ model. 


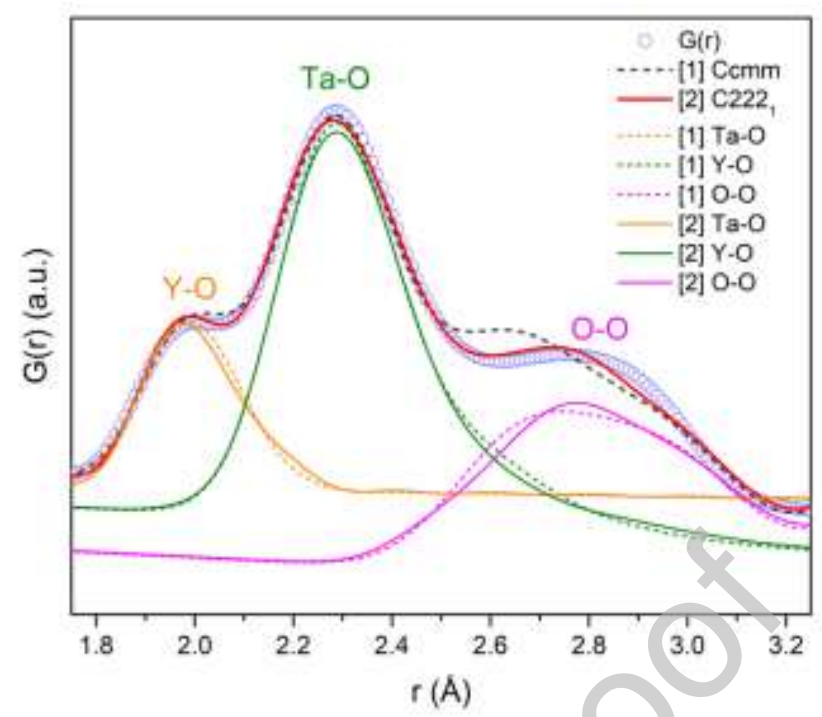

Figure 4: Neutron pair distribution function (PDF) of $\mathrm{Y}_{3} \mathrm{TaO}_{7}$ (blue circles) with refined smallbox models using a Ccmm (dashed black curve) and a $C 222_{1}$ (solid red curve) symmetries. To highlight nearest neighbor bond distances, partial PDFs are shown for both structural model (dashed lines for $\mathrm{Ccmm}$ and solid line for $C 222_{1}$ ). Partial PDFs are shifted for the ease of visualization. 

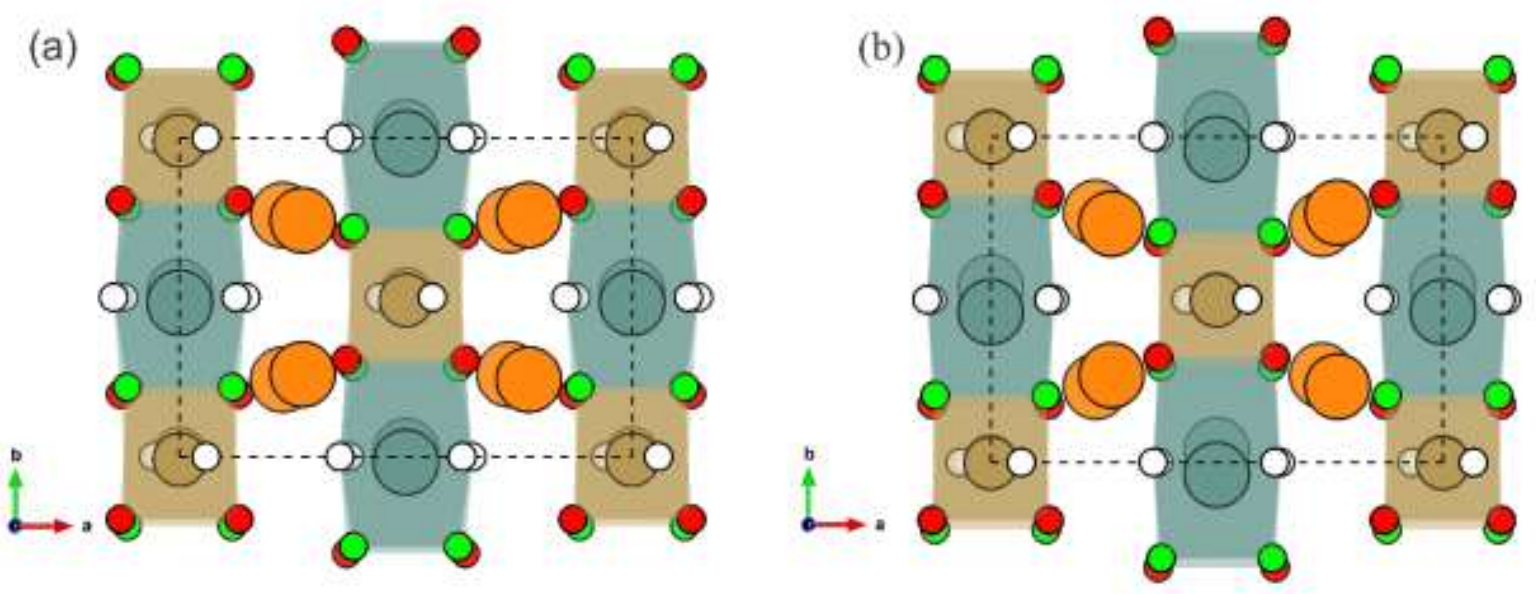

Figure 5: Schematic representations of $\mathrm{Y}_{3} \mathrm{TaO}_{7}$ using a $C 222_{1}$ structural model from (a) experimental data via small-box refinement and (b) DFT calculations. Yttrium cations are shown as cyan spheres (8-coordinated) or as orange spheres (7-coordinated), while tantalum cations are shown as golden sand spheres. Two 8c oxygen sites are shown as red and green spheres while three $4 \mathrm{a}$ oxygen sites are shown as white spheres. Both structural models are shifted by $1 / 4 c$ along [001] for ease of comparison with the Ccmm structure. The dashed black line represents the reference cells. 


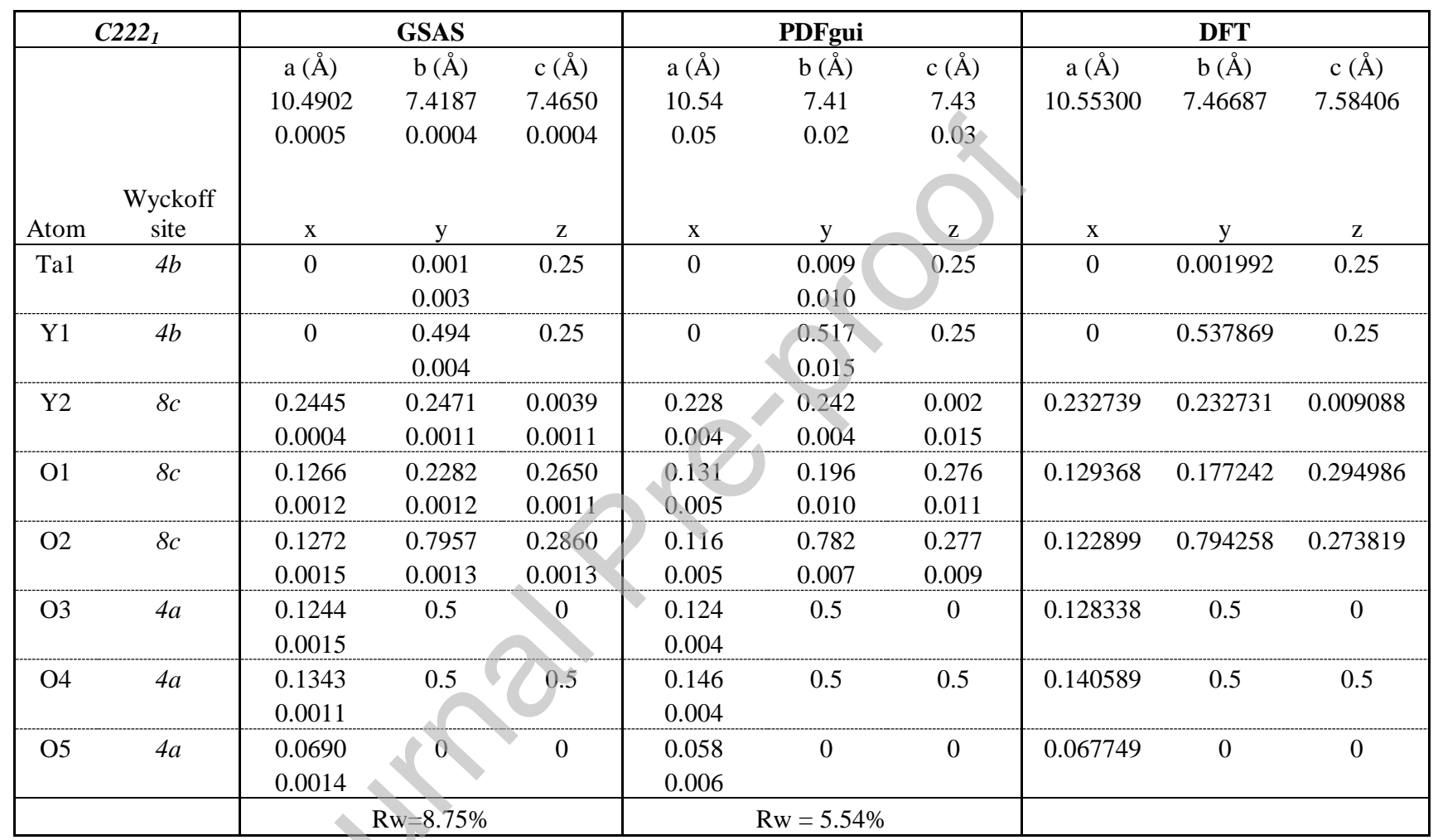




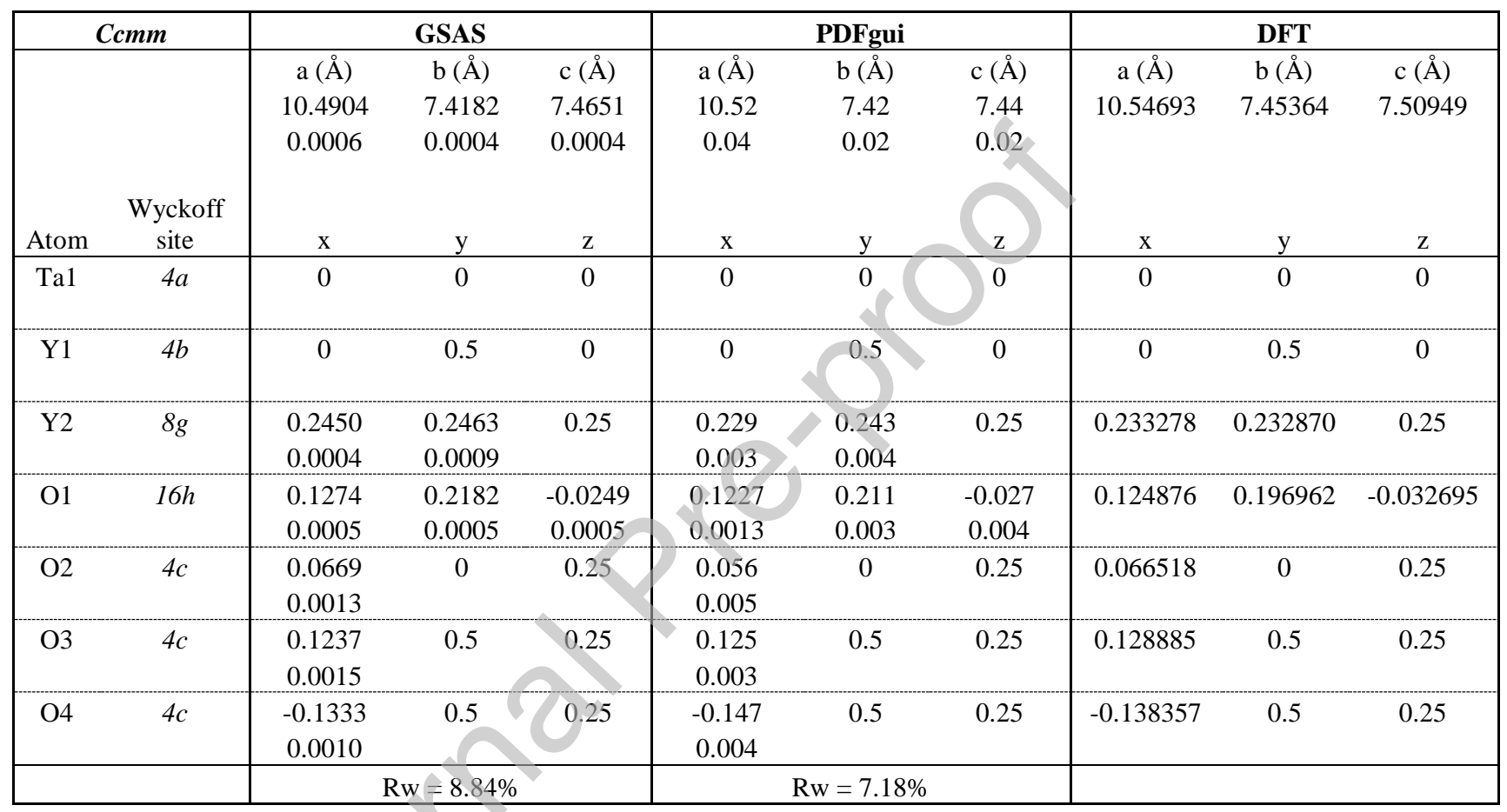

Table 1: Structural information for long- and short-range data refinements along with density functional theory calculations. Unit cell parameters and fractional positional coordinates are followed by sigma values. 


\section{References:}

[1] S. J. Zinkle and G. S. Was, "Materials challenges in nuclear energy," Acta Mater., vol. 61, no. 3 , pp. 735-758, 2013.

[2] J. Lian, R. C. Ewing, F. Zhang, M. Lang, F. Lu, and J. Zhang, "Ion-irradiation-induced structural transitions in orthorhombic Ln2TiO5," Acta Mater., vol. 61, no. 11, pp. 41914199, 2013.

[3] J. Lian, L. M. Wang, K. Sun, and R. C. Ewing, "In situ TEM of radiation effects in complex ceramics," Microsc. Res. Tech., vol. 72, no. 3, pp. 165-181, 2009.

[4] M. Tang et al., "Radiation-induced amorphization resistance and radiation tolerance in structurally related oxides," Nat. Mater., vol. 6, no. 3, pp. 217-223, 2007.

[5] J. Shamblin et al., "Structural response of titanate pyrochlores to swift heavy ion irradiation," Acta Mater., vol. 117, pp. 207-215, 2016.

[6] M. Lang et al., "Review of A2B2O7 pyrochlore response to irradiation and pressure," Nucl. Instruments Methods Phys. Res. Sect. B Beam Interact. with Mater. Atoms, vol. 268, no. 19, pp. 2951-2959, 2010.

[7] L. Thomé et al., "Radiation effects in nuclear materials: Role of nuclear and electronic energy losses and their synergy," Nucl. Instruments Methods Phys. Res. Sect. B Beam Interact. with Mater. Atoms, vol. 307, pp. 43-48, 2013.

[8] J. Shamblin et al., "Similar local order in disordered fluorite and aperiodic pyrochlore structures," Acta Mater., vol. 144, pp. 60-67, 2018.

[9] E. C. O'Quinn et al., "Advanced characterization technique for mechanochemically 
synthesized materials: neutron total scattering analysis," J. Mater. Sci., vol. 53, no. 19, pp. 13400-13410, 2018.

[10] J. Shamblin et al., "Probing disorder in isometric pyrochlore and related complex oxides," Nat. Mater., vol. 15, no. 5, pp. 507-511, 2016.

[11] T. Subramani and A. Navrotsky, "Energetics of Formation and Disordering in Rare Earth Weberite RE3TaO7 Materials," Inorg. Chem., vol. 58, no. 23, pp. 16126-16133, 2019.

[12] M. Wakeshima, H. Nishimine, and Y. Hinatsu, "Crystal structures and magnetic properties of rare earth tantalates $\mathrm{RE}_{3} \mathrm{TaO}_{7}(\mathrm{RE}=$ rare earths $)$, , J. Phys. Condens. Matter, vol. 16, no. 23, pp. 4103-4120, 2004.

[13] F. H. Borges, F. J. Caixeta, R. R. Pereira, S. R. de Oliveira, and R. R. Gonçalves, "Yttrium tantalate containing high concentrations of Eu3+ as dopant: Synthesis and structural and luminescence features," J. Lumin., vol. 199, no. March, pp. 143-153, 2018.

[14] W. T. Fu and D. J. W. IJdo, "On the crystal structures of Ln3MO7 ( $\mathrm{Ln}=\mathrm{Nd}, \mathrm{Sm}, \mathrm{Y}$ and $\mathrm{M}=\mathrm{Sb}$, Ta)-Rietveld refinement using X-ray powder diffraction data,” J. Solid State Chem., vol. 182, no. 9, pp. 2451-2455, 2009.

[15] J. G. Allpress and H. J. Rossell, "Fluorite-related phases Ln3MO7, Ln = rare earth, Y, or Sc, $\mathrm{M}=\mathrm{Nb}$, Sb, or Ta. I. Crystal chemistry,” J. Solid State Chem., vol. 27, no. 1, pp. 105114, 1979.

[16] T. Linda Francis, P. Prabhakar Rao, S. K. Mahesh, T. S. Sreena, and S. Parvathi Babu, "Effect of host structure on the photoluminescence properties of $\mathrm{Ln} 3 \mathrm{TaO} 7: \mathrm{Eu} 3+$ red phosphors," Opt. Mater. (Amst)., vol. 52, pp. 134-143, 2016. 
[17] T. Tanaka, N. Ishizawa, M. Yoshimura, F. Marumo, and H. Oyanagi, "Exafs analysis and reinvestigation of the structure of a defect-fluorite-type compound, y3tao7," J. Solid State Chem., vol. 114, no. 1, pp. 79-87, 1995.

[18] G. King, C. M. Thompson, J. E. Greedan, and A. Llobet, "Local structure of the vacancy disordered fluorite $\mathrm{Yb} 3 \mathrm{TaO} 7$ from neutron total scattering," J. Mater. Chem. A, vol. 1, no. 35, pp. 10487-10494, 2013.

[19] J. Neuefeind, M. Feygenson, J. Carruth, R. Hoffmann, and K. K. Chipley, “The Nanoscale Ordered MAterials Diffractometer NOMAD at the Spallation Neutron Source SNS," Nucl. Instruments Methods Phys. Res. Sect. B Beam Interact. with Mater. Atoms, vol. 287, pp. 68-75, 2012.

[20] H. M. Rietveld, "A profile refinement method for nuclear and magnetic structures," $J$. Appl. Crystallogr., vol. 2, no. 2, pp. 65-71, 2002.

[21] B. H. Toby, "EXPGUI, a graphical user interface for GSAS," J. Appl. Crystallogr., vol. 34, no. 2, pp. 210-213, Apr. 2001.

[22] E. S. Božin et al., "PDFfit2 and PDFgui: computer programs for studying nanostructure in crystals,"J. Phys. Condens. Matter, vol. 19, no. 33, p. 335219, 2007.

[23] J. P. Perdew, K. Burke, and M. Ernzerhof, "Generalized gradient approximation made simple," Phys. Rev. Lett., vol. 77, no. 18, pp. 3865-3868, 1996.

[24] G. Kresse and J. Furthmüller, "Efficiency of ab-initio total energy calculations for metals and semiconductors using a plane-wave basis set," Comput. Mater. Sci., vol. 6, no. 1, pp. 15-50, 1996. 
[25] W. C. Hamilton, " Significance tests on the crystallographic R factor ," Acta Crystallogr., vol. 18 , no. 3, pp. 502-510, 1965. 


\section{Declaration of interests}

$\bigotimes$ The authors declare that they have no known competing financial interests or personal relationships that could have appeared to influence the work reported in this paper.

$\square$ The authors declare the following financial interests/personal relationships which may be considered as potential competing interests: 


\section{Graphical Abstract}
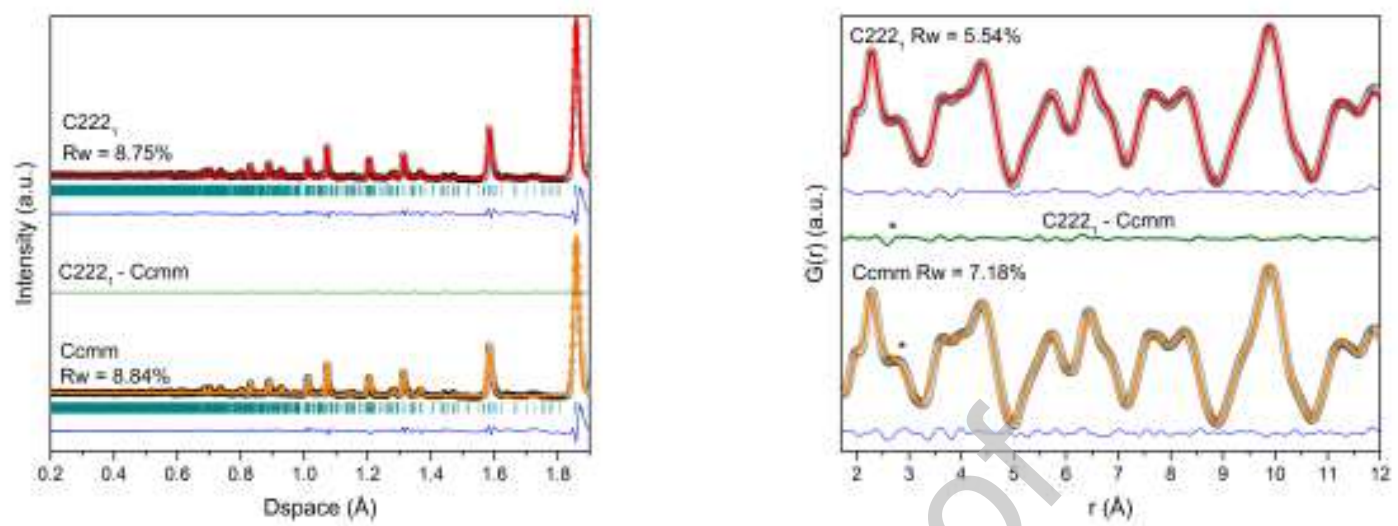